\title{
Cholinergic basal forebrain neurons regulate fear extinction consolidation through p75 neurotrophin receptor signaling
}

\author{
Zoran Boskovic ${ }^{1,2,3}$, Michael R. Milne ${ }^{1,2,3}$, Lei Qian (10 ${ }^{1,2}$, Hamish D. Clifton ${ }^{1,4}$, Alice E. McGovern ${ }^{3}$, Marion T. Turnbull ${ }^{1,2,3}$,
} Stuart B. Mazzone ${ }^{3}$ and Elizabeth J. Coulson (10,2,3

\begin{abstract}
Cholinergic basal forebrain (CBF)-derived neurotransmission plays a crucial role in regulating neuronal function throughout the cortex, yet the mechanisms controlling cholinergic innervation to downstream targets have not been elucidated. Here we report that removing the p75 neurotrophin receptor ( $\mathrm{p} 75^{\mathrm{NTR}}$ ) from $\mathrm{cBF}$ neurons induces a significant impairment in fear extinction consolidation. We demonstrate that this is achieved through alterations in synaptic connectivity and functional activity within the medial prefrontal cortex. These deficits revert back to wild-type levels upon re-expression of the active domain of $p 75^{\mathrm{NTR}}$ in adult animals. These findings demonstrate a novel role for cholinergic neurons in fear extinction consolidation and suggest that neurotrophic signaling is a key regulator of cholinergic-cortical innervation and function.
\end{abstract}

\section{Introduction}

Cholinergic basal forebrain (cBF) neurons play an important role in cognition through their modulation of cortical neurotransmission ${ }^{1,2}$. These neurons have a significant influence on the activity of neuronal populations within a variety of cortical and subcortical areas, including the entorhinal cortex ${ }^{3-5}$, neocortex ${ }^{6}$, visual cortex $^{7}$, striatum $^{8}$, hippocampus ${ }^{9}$ and motor cortex ${ }^{10,11}$, with cholinergic dysfunction underpining cognitive impairment in a number of neurodegenerative and mental health conditions ${ }^{12-14}$.

One of the defining features of $\mathrm{cBF}$ neurons is that they comprise one of the few populations in the adult brain

\footnotetext{
Correspondence: Elizabeth J. Coulson (e.coulson@uq.edu.au)

${ }^{1}$ Centre for Ageing Dementia Research, The University of Queensland, Brisbane, QLD 4072, Australia

${ }^{2}$ Queensland Brain Institute, The University of Queensland, Brisbane, QLD 4072, Australia
}

Full list of author information is available at the end of the article. that expresses the p75 neurotrophin receptor $\left(\mathrm{p} 75^{\mathrm{NTR}}\right)$. We and others have shown that mice that completely lack $\mathrm{p} 75^{\mathrm{NTR}}$ from conception or those in which $\mathrm{p} 75^{\mathrm{NTR}}$ expression is removed from $\mathrm{CBF}$ neurons from postnatal day 4 have altered $\mathrm{CBF}$ neuronal innervation to the cortex, which correlates with improvements in spatial navigation, indicative of enhanced memory ${ }^{15-19}$. These data suggested that the function of cortical neurons is regulated by the extent of $\mathrm{cBF}$ innervation to target regions, which is mediated through the actions of p $75^{\mathrm{NTR}}$ signaling. However, it is unclear whether $\mathrm{p} 75^{\mathrm{NTR}}$ plays a role in axonal innervation and cortical function during adult life rather than development.

Recently, it has been suggested that cBF neurons are involved in regulating the response to aversive stimuli, with fear conditioning being regulated by nucleus basalis of Meynert cBF innervation of the amygdala, and lesionining of medial septal $\mathrm{cBF}$ neurons impairing the acquisition of fear extinction-the process whereby fearful

\section{(c) The Author(s) 2018}

(c) (i) Open Access This article is licensed under a Creative Commons Attribution 4.0 International License, which permits use, sharing, adaptation, distribution and reproduction cc) in any medium or format, as long as you give appropriate credit to the original author(s) and the source, provide a link to the Creative Commons license, and indicate if changes were made. The images or other third party material in this article are included in the article's Creative Commons license, unless indicated otherwise in a credit line to the material. If material is not included in the article's Creative Commons license and your intended use is not permitted by statutory regulation or exceeds the permitted use, you will need to obtain permission directly from the copyright holder. To view a copy of this license, visit http://creativecommons.org/licenses/by/4.0/. 


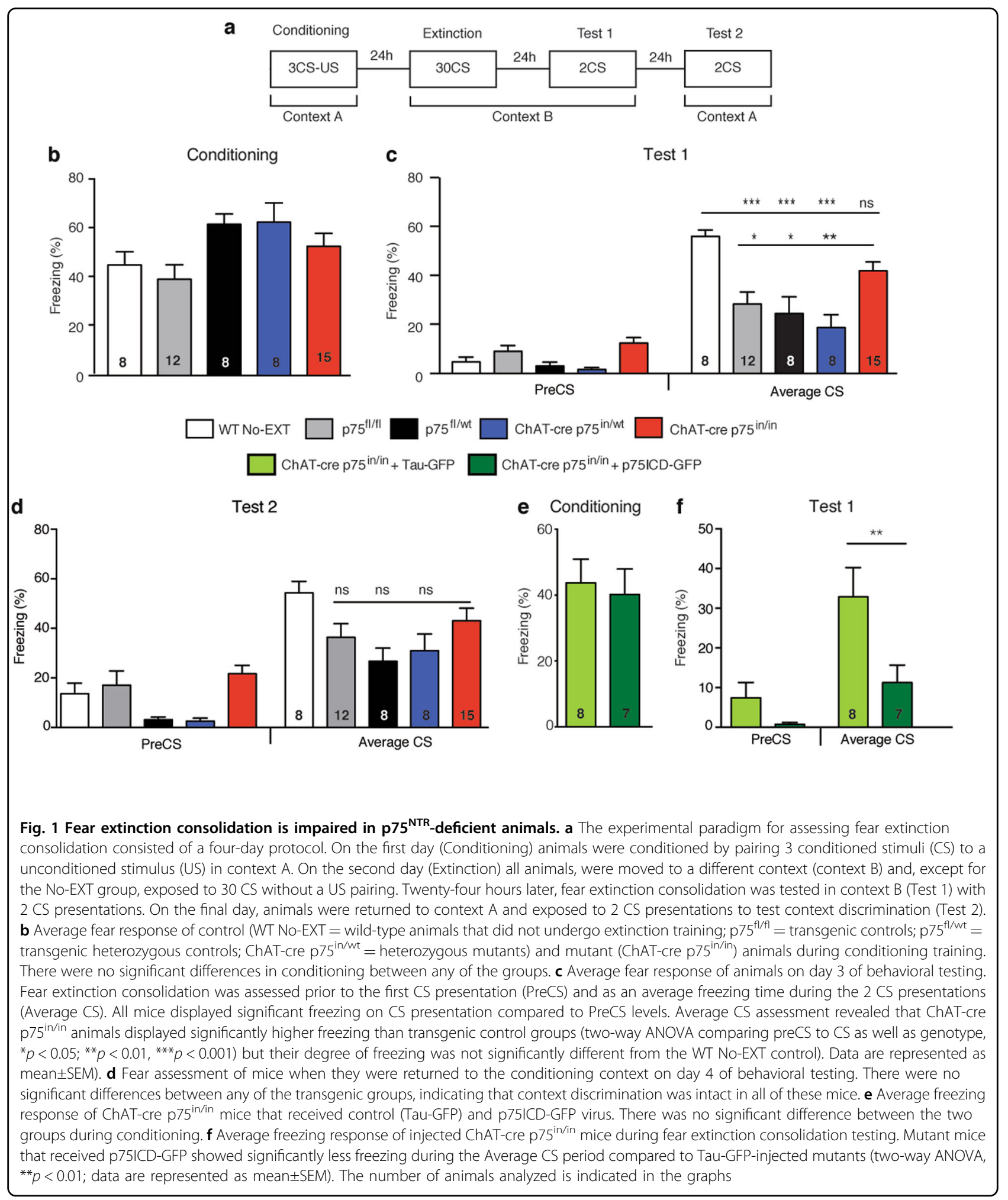

responses can be diminished ${ }^{20-22}$. Compared with spatial navigation, the neural circuits and behavioral paradigms associated with fear conditioning and extinction are more clearly defined, allowing $\mathrm{cBF}$ neuronal function to be interrogated. In fear extinction paradigms, a conditioned stimulus (CS), such as a tone, is paired with an aversive unconditioned stimulus (US) such as a footshock, leading to a long-lasting response to the $\mathrm{CS}^{23}$. This response is 
diminished through fear extinction by subsequent repeated CS presentations that are not paired with the US. While the hippocampus provides context encoding for fear learning and extinction ${ }^{24}$, it is the infralimbic prefrontal cortex (ILPFC) within the medial prefrontal cortex (mPFC) that is critical for fear extinction consolidation $^{25,26}$.

We hypothesized that, if $\mathrm{p} 75^{\mathrm{NTR}}$ played a role in adult cBF connectivity and function, we would observe a phenotype in adult conditional p75 ${ }^{\mathrm{NTR}}$ knockout mice $\left(\right.$ ChAT-cre $\left.\mathrm{p} 75^{\mathrm{in} / \mathrm{in}}\right)$, which would be rescued by acute reexpression of $\mathrm{p} 75^{\mathrm{NTR}}$ within $\mathrm{cBF}$ neurons. We therefore tested these mice in fear conditioning/extinction paradigms and measured the $\mathrm{CBF}$ axonal and synaptic arborization within the relevant circuit.

\section{Materials and methods}

\section{Animals}

The $\mathrm{p} 75^{\mathrm{f} / \mathrm{fl}}$ conditional knockout mouse ${ }^{16}$ and the choline-acetyltransferase (ChAT)-IRES-cre $\operatorname{strain}^{27}$ have been described previously. All animals used were 2-4 months of age. All behavioral studies were performed using male mice. Neuronal tracing studies were performed using both males and females. Mice were maintained on a 12-hour light/dark cycle (lights on at 7:00 a. $\mathrm{m}$.), with food and water provided ad libitum in specific pathogen free (SPF) OptiMouse caging. All procedures were approved by the University of Queensland Animal Ethics Committee and conducted in accordance with the Australian Code of Practice for the Care and Use of Animals for Scientific Purposes.

\section{Behavioral tasks}

Fear extinction was assessed using a previously described protocol ${ }^{26}$ (Fig. 1a). Two contexts (A and B) were used. Both conditioning chambers (Coulbourn Instruments) had two transparent walls and stainless steel grid floors $(3.2 \mathrm{~mm}$ in diameter, $8 \mathrm{~mm}$ appart); however, the floors in context B were covered with flat white acrylic inserts to minimize context generalization. Context A had a lemon-scented odor and context B had a vinegarscented odor. Individual digital cameras were mounted in the ceiling of each chamber and connected via a quad processor for automated scoring of freezing (Freezeframe). Fear conditioning was induced in context A. Mice received $3 \mathrm{CS} / \mathrm{US}$ pairings at equal intervals over $14 \mathrm{~min}$. The CS was a $120 \mathrm{~s}$ white noise stimulus at $80 \mathrm{~dB}$ whereas the US was a $0.5 \mathrm{~mA}, 1 \mathrm{~s}$ footshock. Twenty-four hours later, animals underwent extinction training in context B over 65 min. A $120 \mathrm{~s}$ PreCS period was followed by 30 unpaired CS of $120 \mathrm{~s}$ with $5 \mathrm{~s}$ between tones finishing with $120 \mathrm{~s}$ of silence. Mice that did not undergo extinction training were placed in context B but did not receive any CS presentations. The extinction test was a further $24 \mathrm{~h}$ later in context B, where they were exposed to two unpaired CS. Finally, to measure contextual fear memory animals were returned to context $\mathrm{A}$, where they were exposed to 2 unpaired CS. Animals with freezing below 10 percent on the conditioning day were excluded from the analysis. The data for freezing prior to the first CS (PreCS) and the average freezing during both CS presentations (Average CS) during testing are provided in the results.

\section{Viruses}

Tau-GFP (used to assess cholinergic innervation) and p $75^{\text {NTR }}$ intracellular domain-green fluorescent fusion protein (p75ICD-GFP; used for rescue experiments) expression constructs were packaged into a pseudotype adeno-associated virus (AAV) 2/1 chimeric vector for in vivo delivery into the mice. Each cDNA was inserted into the DIO Cre-ON plasmid in reverse orientation, which was driven by the ubiquitous promoter elongation factor-1 alpha (EF1 $\alpha$ ) and was flanked by oppositely oriented loxP and lox2272 sites (pAAV-EF1a-DIO). In the presence of cre recombinase, the transgene was expres$\mathrm{sed}^{28}$. The AAV pseudotyped vectors were generated as previously described $^{29}$. The trans-synaptic HSV129 tracer virus has also been described previously ${ }^{30}$.

\section{Surgical procedures}

Animals were anesthetized by intraperitoneal (i.p.) injection of ketamine $(100 \mathrm{mg} / \mathrm{kg})$ and the muscle relaxant xylazine $(10 \mathrm{mg} / \mathrm{kg})$. Each mouse was then placed in a stereotaxic frame (David Kopf Instruments) and the skull exposed. Viral infusions were performed using a $30 \mathrm{G}$ infusing system using a guide and internal cannula (Plastics1), directly into the medial septum (the needle was lowered into the medial septum (A-P $0.9 \mathrm{~mm} ; \mathrm{M}-\mathrm{L}$ $0 \mathrm{~mm}$; D-V $4.2 \mathrm{~mm}$ from Bregma) where either undiluted HSV129 virus $(0.5 \mu \mathrm{l})$, Tau-GFP $(1 \mu \mathrm{l})$ or p75ICD-GFP $(1 \mu \mathrm{l})$ was infused. For all procedures, the internal cannula was left in place for $8 \mathrm{~min}$ to allow for diffusion before removal. Immediately after surgery, mice were injected subcutaneously with the analgesic torbugesic $(2 \mathrm{mg} / \mathrm{kg})$, and the antibiotic Baytril $(5 \mathrm{mg} / \mathrm{kg})$ and allowed to recover before being returned to their home cage where they were housed two per cage but kept separated by a clear Perspex barrier that allowed aural and olfactory exchange. Mice were 8-12 weeks old at the time of injection and were left for 1 month (Tau-GFP and p75ICD-GFP) or 48-72 h (HSV129) to allow for viral expression before behavioral testing/killing.

\section{Histological tissue preparation}

Brains from $4 \%$ formaldehyde-perfused mice were postfixed overnight, preserved in $20 \%$ sucrose solution for $24 \mathrm{~h}$, and embedded in O.C.T compound (Tissue-Tek). 
Forty $\mu \mathrm{m}$ coronal sections were cut in three serially adjacent sets through the basal forebrain and prefrontal cortex using a sliding microtome (SM2000r, Leica). Brains used for phosphorylated extracellular signal-regulated kinase (pERK) quantification were from naïve mice or those euthanized not more than $1 \mathrm{~h}$ after the fear extinction test.

\section{Immunohistochemistry}

For immunofluorescence labeling, free-floating $40 \mu \mathrm{m}$ sections were immunostained using goat anti-GFP (1:1000, Abcam ab5450), rabbit anti-DsRed (1:200; Clontech 632496), rabbit anti-pERK (1:200; Millipore 05$797 \mathrm{R})$ and mouse anti-CamKII (1:200; Abcam ab22609). Sections were mounted onto slides and coverslipped using fluorescence mounting medium (Dako S3023).

To measure neuronal activity, labeling of pERK was visualized using rabbit anti-pERK antibody (1:1000; Millipore 05-797 R), biotinylated donkey anti-rabbit IgG (1:1000; Abcam ab97062) and ABC reagent (Vector Elite kit: Vector Laboratories PK-6100). Black immunoreactive cytoplasm labeled for pERK was revealed by a nickelintensified diaminobenzidine reaction and slices were coverslipped with DePeX (Sigma-Aldrich 06522).

\section{Histological quantifications}

All cell counts and quantifications for the mPFC were performed on sections between $2.3 \mathrm{~mm}$ anterior to bregma and $1.5 \mathrm{~mm}$ anterior to bregma, medial septum/ diagonal band of Broca sections were between $1.3 \mathrm{~mm}$ anterior to bregma and $0.1 \mathrm{~mm}$ anterior to bregma and hippocampal sections from between $1.2 \mathrm{~mm}$ posterior to bregma and $2.2 \mathrm{~mm}$ posterior to bregma. Sections from these regions were randomly selected and the researcher was blind to the group allocation. When quantifying subregions of the $\mathrm{mPFC}$, immunohistological image of the section being analyzed was overlayed with the Allen brain atlas demarcations on a computer at a high magnification, with stereological rules being applied when cells were touching a line.

\section{Neurite density}

To measure cholinergic innervation in the MPFC and hippocampus on a per cell basis, Tau-GFP and p75ICDGFP virus-infused animals were euthanized following a 6-week incubation period and the extent of GFP labeling was used as a proxy for density of cBF neuronal innervation. First, the number of GFP-positive cell bodies in every third section at the injection site was counted. Then 3-6 mPFC sections were randomly selected from each brain and stained for GFP and imaged. If fewer than 10 cells per section were infected, or there were no obvious GFP-positive axons in the target areas the animals were excluded from the quantification The images were converted to an 8-bit black and white image for analysis using ImageJ v1.49 (NIH). A region of interest (ROI) was drawn around the mPFC/hippocampal area of each section aligned to the atlas of Franklin and Paxinos (2007). Threshold settings were used to subtract background staining and artifacts from the image. The mean pixel density within the ROI was then calculated and averaged for each mouse to provide the average neurite density in the $\mathrm{mPFC} /$ hippocampus. These data were then converted to average density per infected neuron at the injected site for each mouse by dividing the neurite density by the number of labeled neurons. The average ratio was compared between genotypes.

\section{Synaptic connectivity}

To measure the extent of synaptic connectivity from the medial septum to the mPFC and hippocampus, the number of HSV129 GFP- and tdTomato-labeled neurons in the mPFC (6-8 randomly selected sections per animal) and hippocampus (7-10 randomly selected sections per animal) were counted following tissue processing and immunohistochemistry.

\section{Imaging}

Imaging was performed on an Axio Imager upright fluorescence microscope (Zeiss) and Metafer VSlide Scanner (Metasystems) using Zeiss Axio Imager Z2. All measurements and analyses were performed using Imaris 7.2.3 software (Bitplane).

\section{Statistics}

Results are expressed as mean \pm standard error of mean. Statistical analyses were conducted using two-tailed student's t-tests, one-way ANOVA or two-way ANOVA (CS one factor and genotype second factor) with Sidak's posthoc test (Graphpad Prism 6) with the significance threshold set at $p<0.05$.

\section{Results \\ p $75^{\text {NTR }}$ is required for successful fear extinction consolidation}

To determine the effects of $\mathrm{p} 75^{\mathrm{NTR}}$ removal from cBF neurons on fear extinction, we subjected two cohorts of ChAT-cre $775^{\mathrm{in} / \mathrm{in}}$ mice (mice in which the floxed allele was inverted-in/in-in cholinergic neurons) and littermate heterozygous and cre-negative controls ${ }^{16}$ to a fourday, strong extinction paradigm (Fig. 1a-c). Mice of all genotypes showed similar freezing behavior following conditioning training on day $1(\mathrm{~F} \quad(4,46)=0.756$, $p=0.5592$ ). Similarly, we observed no difference between groups during extinction training on the following day (data not shown), with all mice showing $40 \%$ freezing on first CS presentation compared to preCS levels (CS factor: $\mathrm{F}(1,92)=132.2 ; p<0.0001)$, and freezing decreased over 
10 CS presentations $(\mathrm{F}(10,275)=3.305, p=0.0005)$. However, there was no difference in freezing response between genotypes (time/genotype interaction $\mathrm{F}(10,275)=0.6975, p=0.7267)$. Conversely, during the extinction test on the third day, although all mice froze significantly on first CS presentation compared to preCS levels (CS factor: $\mathrm{F}(1,92)=132.2 ; p<0.0001)$, there was a significant interaction of genotype in response to the CS $(\mathrm{F}(4,92)=5.917, p=0.0003)$, with $\mathrm{p} 75^{\mathrm{NTR}}$-knockout mice showing significantly greater freezing than crenegative homozygous $\left(\mathrm{p} 75^{\mathrm{fl} / \mathrm{fl}} ; p=0.036\right)$ and heterozygous controls ( $\mathrm{p} 75^{\mathrm{fl} / \mathrm{wt}} ; p=0.011$ ), as well as heterozygous mutants (ChAT-cre p75 $5^{\text {in } / \mathrm{wt}} ; \quad p=0.0002$ ). Furthermore, the freezing behavior of ChAT-cre $\mathrm{p} 75^{\mathrm{in} / \mathrm{in}}$ animals was not significantly different from that of wildtype animals which underwent conditioning but not extinction training. Nonetheless, when returned to the original conditioning context on day 4 , there were no significant differences in the levels of freezing between ChAT-cre $\mathrm{p} 75^{\mathrm{in} / \mathrm{in}}$ animals, littermate $\mathrm{p} 75^{\mathrm{fl} / \mathrm{fl}}$ controls and heterozygous controls that had undergone extinction training (Fig. 1d; CS/genotype interaction F $(4,92)=$ 1.353, $p=0.2566$ ). This indicates that the deficit in the p $75^{\mathrm{NTR}}$-knockout animals is specific to consolidation of fear extinction rather than any other aspect of fear-related behavior.

Recombination of the $\mathrm{p} 75^{\mathrm{NTR}}$ allele in ChAT-cre $\mathrm{p} 75^{\mathrm{in} / \mathrm{in}}$ animals prevents $\mathrm{p} 75^{\mathrm{NTR}}$ expression from postnatal day $4^{16}$. Hence, the observed behavioral change could be a consequence of differences in postnatal development (reduced programmed cell death resulting in a $\sim 25 \%$ increase in adult cBF neurons ${ }^{16}$ ) or the result of an acute role for $\mathrm{p} 75^{\mathrm{NTR}}$ in adult neurons. The intracellular domain of $\mathrm{p} 75^{\mathrm{NTR}}$ (p75ICD) has been identified as a critical mediator of $\mathrm{p} 75^{\mathrm{NTR}}$ function ${ }^{31}$. This fragment lacks the ligand-binding and transmembrane domains but is considered to be a constitutively activated form of p75 $5^{\text {NTR32 }}$ as it can mediate both axonal pruning ${ }^{33}$ and axonal growth, in the latter case by facilitating Trk receptor signaling ${ }^{31,34}$. The p75ICD-GFP AAV, or a control AAV containing the identical genetic backbone but expressing a benign GFP-tagged tau protein (Tau-GFP; Supplementary Figure S1a, b), was injected into the medial septum of the basal forebrain in adult ChAT-cre $\mathrm{p} 75^{\text {in/in }}$ animals. Following a 6-week period to allow for robust expression of the virus $(97 \pm 0.71 \%$ of medial septum ChAT-positive cells expressed GFP; data not shown), the AAV-injected mutant animals were subjected to the same fear conditioning/extinction paradigm. Locomotion and exploratory behavior were not affected by p75ICD-GFP expression (Supplementary Figure S1c, d), and no differences were observed during conditioning training (Fig. 1e). However, p75ICD-GFP-injected ChAT-cre p $75^{\text {in/in }}$ animals displayed a significant decrease in freezing during the extinction test compared to control-injected ChAT-cre p75 ${ }^{\text {in/in }}$ animals (Fig. 1f; Treatment factor $\mathrm{F}(1,26)=13.08$; $p=0.0013$ ), possibly freezing less than $\mathrm{p} 75^{\mathrm{fl} / \mathrm{fl}}$ animals (c.f. Fig. 1c). These results demonstrate that medial septum $\mathrm{cBF}$ neurons are important for regulating the consolidation of fear extinction and that $\mathrm{p} 75^{\mathrm{NTR}}$ signaling in the adult $\mathrm{cBF}$ neurons is involved in this process.

\section{p75 ${ }^{\mathrm{NTR}}$ has a negligible effect on the extent of innervation of cholinergic neurons to the PFC}

The observed behavioral changes in ChAT-cre $\mathrm{p} 75^{\mathrm{in} / \mathrm{in}}$ animals could be due to alterations in cBF innervation of downstream cortical targets that are involved in fear extinction. We have previously demonstrated that ChATcre $\mathrm{p} 75^{\mathrm{in} / \mathrm{in}}$ animals have increased cholinergic innervation of the barrel cortex but not the hippocampus ${ }^{16}$. However, we did not determine whether this phenotype was due to the $25 \%$ increase in the number of cBF neurons, or whether individual $\mathrm{CBF}$ neurons have increased arborization in the absence of $\mathrm{p} 75^{\mathrm{NTR}}$. To investigate this, we injected the cre recombinase-dependent Tau-GFP AAV into the medial septum of control (ChAT-cre) and ChAT-cre $\mathrm{p} 75^{\mathrm{in} / \mathrm{in}}$ animals, thereby labeling cBF axons. Brain structures known to be important in fear extinction consolidation include the amygdala, hippocampus and $\mathrm{mPFC}^{1,25,35}$. Medial septum $\mathrm{cBF}$ neurons project to the latter two regions but not the amygdala, which is innervated by the nucleus basalis of Meynert cBF neurons. We therefore quantified the density of GFP-labeled cBF axons in the hippocampus and mPFC relative to the number of medial septum $\mathrm{CBF}$ neurons infected with the virus. No significant difference was observed between mutant and control animals in terms of the extent of GFP labeling in either the hippocampus (Supplementary Figure S2a, b) or the mPFC (Fig. 2a, b).

We also assessed cBF axonal innervation in the PFC and hippocampus in control and ChAT-cre $\mathrm{p} 75^{\mathrm{in} / \mathrm{in}}$ mice expressing p75ICD-GFP by quantifying the extent of axonal GFP staining. Expression of p75ICD-GFP in p $75^{\mathrm{NTR}}$-deficient cBF neurons had no significant effect on hippocampal (Supplementary Figure S2c, d) or mPFC innervation in comparison to its effect on wild-type cBF neurons (Fig. 2c, d). This indicates that re-expression of p75ICD does not restore fear extinction by mediating axonal pruning.

\section{p75 ${ }^{\mathrm{NTR}}$ regulates basal forebrain connectivity to the PFC}

As $\mathrm{cBF}$ neurons can regulate neurotransmission by both volume release and synaptic release ${ }^{2}$, we next investigated whether $\mathrm{p} 75^{\mathrm{NTR}}$ expression regulates synaptic connections between $\mathrm{CBF}$ neurons and the mPFC. This was achieved by using an anterograde trans-synaptic Herpes simplex virus (HSV129; Supplementary Figure S3a) to label the postsynaptic neurons innervated by cholinergic and/or noncholinergic basal forebrain synaptic efferents ${ }^{30}$. This virus 

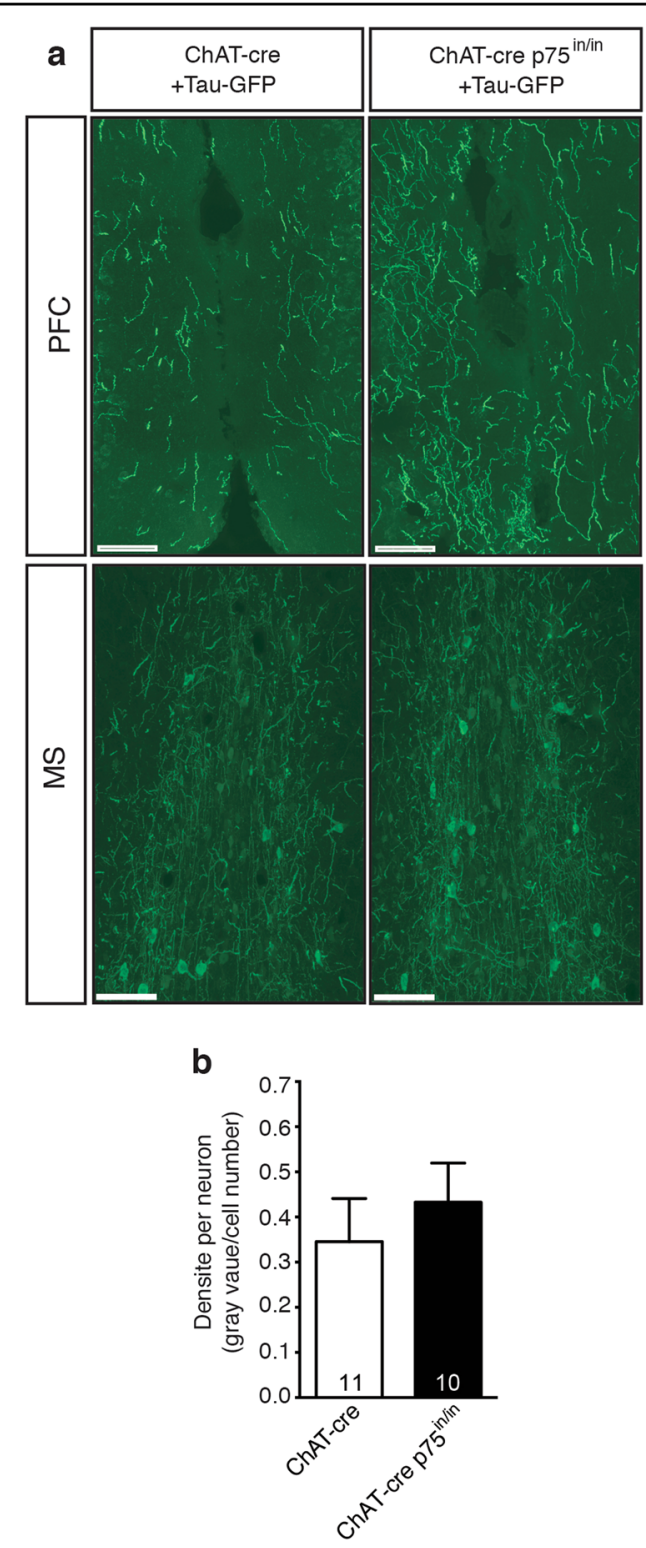
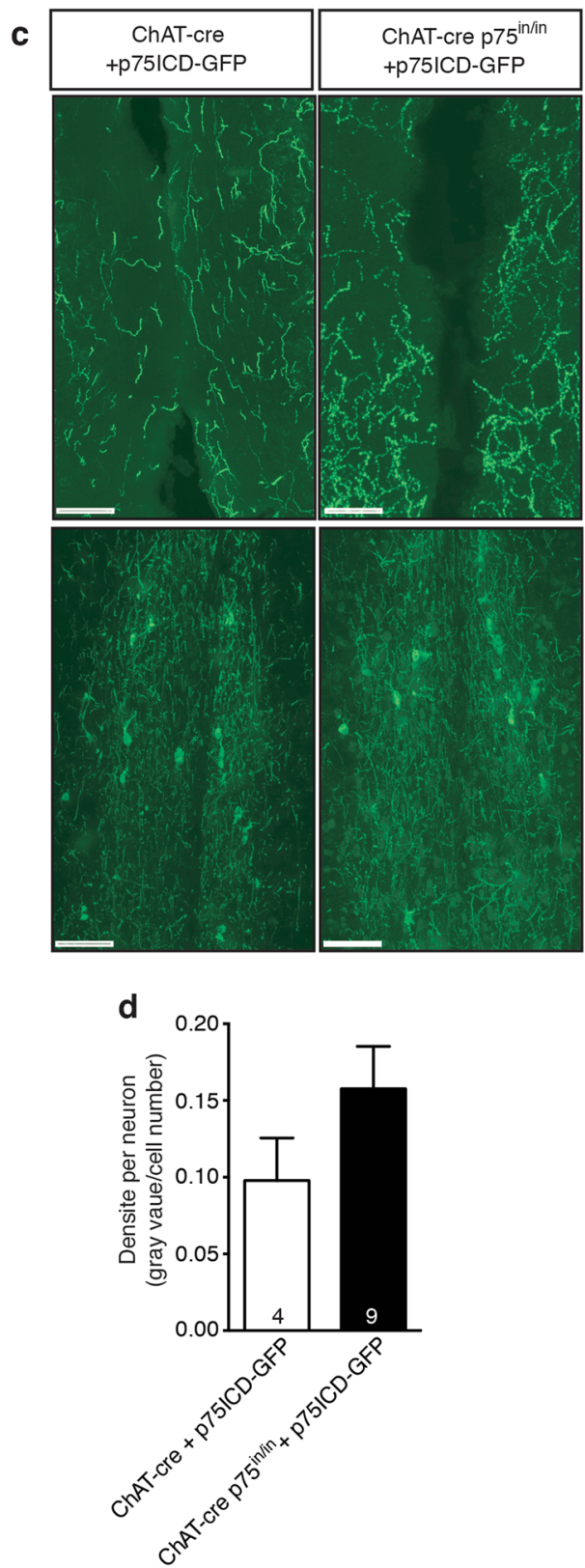

Fig. 2 Arbour size of individual neurons is unaffected by the absence of $\mathbf{p} 75^{\mathrm{NTR}}$. a Representative images of the prefrontal cortex (PFC; target) and medial septum (MS; injection site) and of cre-expressing control (ChAT-cre) and mutant (ChAT-cre p $75^{\text {in/in }}$ ) mice injected with Tau-GFP. Sections were labeled using an anti-GFP antibody (green). Scale bar $=100 \mu \mathrm{m}$. b Average GFP-labeled axonal density per infected cell of mice injected with Tau-GFP AAV. No significant difference in axonal density/cBF neuron was observed between control and mutant mice. $\mathbf{c}$ Representative images of CBF axonal innervation of the PFC and infected cells in the MS of control and mutant mice injected with p75ICD-GFP. Sections were labeled using an anti-GFP antibody (green). d Average GFP-labeled axonal density per infected cell of animals injected with p75ICD-GFP AAV. No significant effect of the p75ICD-GFP injections were observed in animals of either genotype. The number of animals analyzed is indicated in the graphs

expresses GFP in non-cre recombinase-expressing (noncholinergic) cells, but undergoes recombination in the cre recombinase-expressing cholinergic cells, leading to the expression of a TdTomato reporter. The native or recombined virus is then passed trans-neuronally to postsynaptic neurons. Viral transmission was limited by sacrificing the animals $72 \mathrm{~h}$ after viral injection ${ }^{36}$, and the number of HSV129-labeled cells in the MPFC and hippocampus were counted. The number of TdTomato-expressing cells in the mPFC of mutant animals was significantly ( 10-fold) higher 

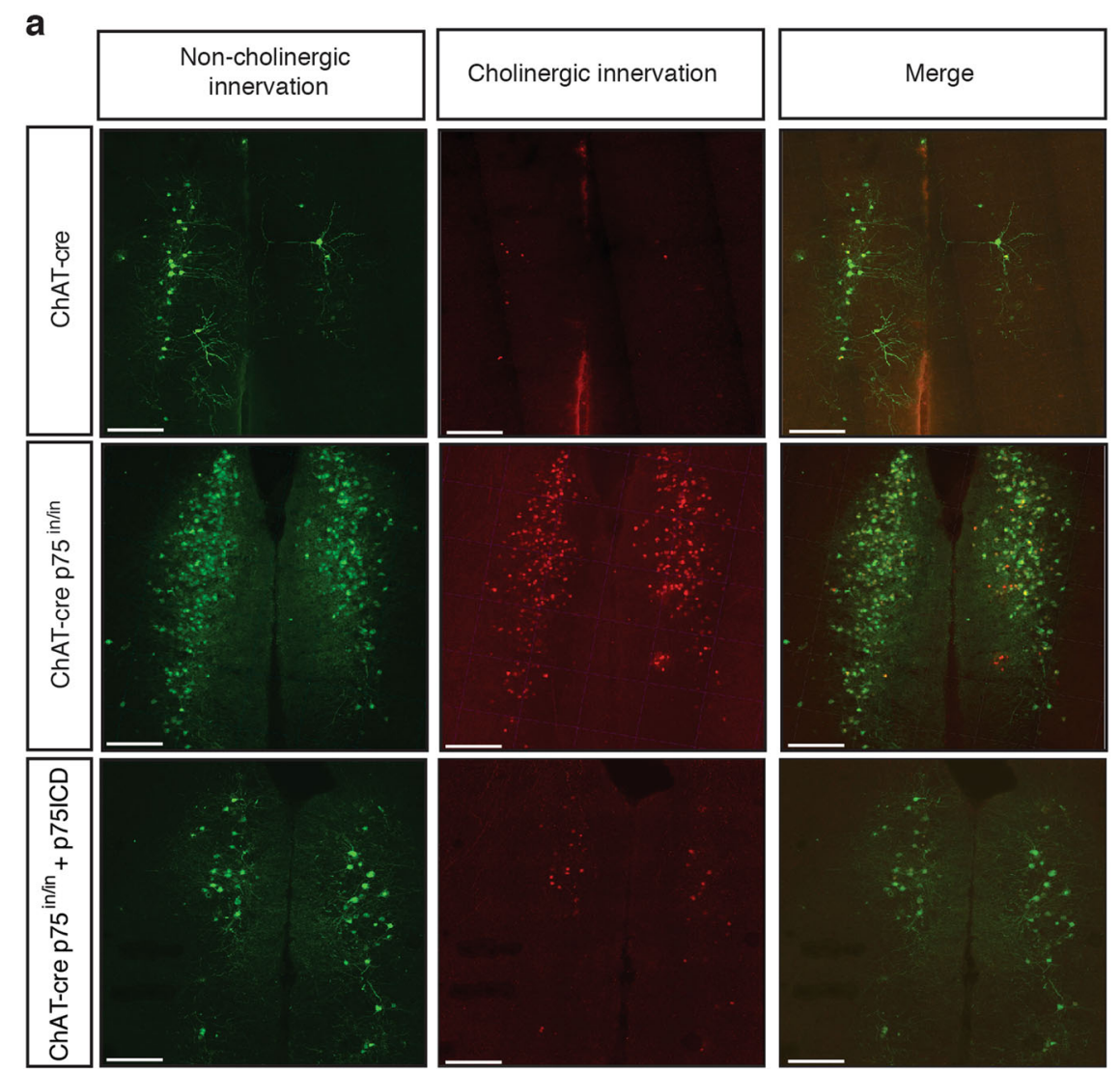

b

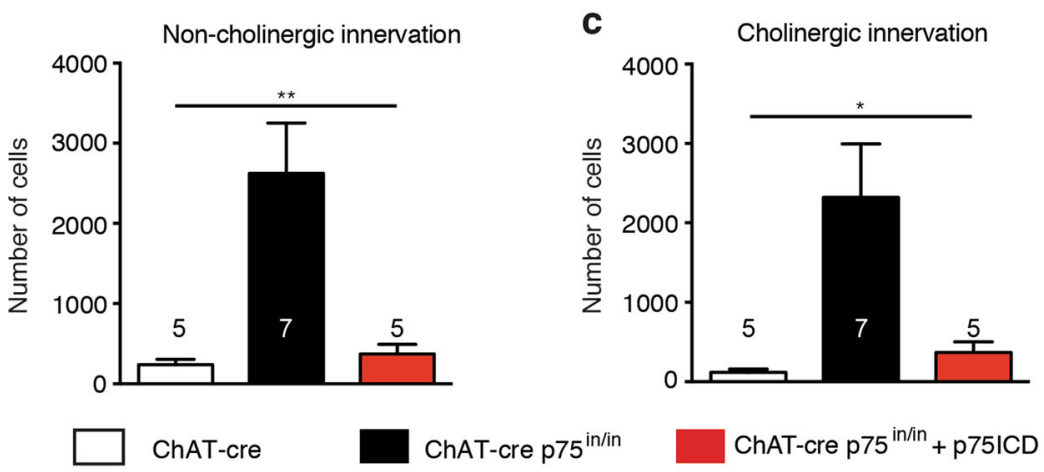

Fig. 3 p75 ${ }^{\text {NTR }}$ signalling affects synaptic connectivity of CBF neurons. a Representative images of the prefrontal cortex (PFC) of cre-expressing controls (ChAT-cre), mutants (ChAT-cre p75 $5^{\text {in/in }}$ ) and mutants injected with p75ICD-GFP. Postsynaptic cells in the PFC innervated by non-cholinergic basal forebrain neurons (green) were labeled against the GFP-expressing version of HSV129 virus (See Supplementary Figure S3A). Cells innervated by CBF neurons (red) were labeled against the tdTomato-expressing version of the HSV129 virus. Scale bar $=200 \mu \mathrm{m}$. $\mathbf{b}$ There was a significant increase in GFP labeling in the PFC of ChAT-cre p $75^{\text {in/in }}$ mutants compared to control and p75ICD-injected mutant mice (one-way ANOVA, ${ }^{* *} p<0.01$; the data are represented as mean \pm SEM). $\mathbf{c}$ A significant increase in tdTomato labeling was also observed in the PFC of ChAT-cre p75 ${ }^{\text {in/in }}$ animals compared to controls, which reverted back to control levels upon injection of p75ICD (one-way ANOVA, ${ }^{*} p<0.05,{ }^{* *} p<0.01$; data are represented as mean \pm SEM)

than that found in control ChAT-cre animals (Fig. 3a-c), indicating that the number of synapses per cholinergic basal forebrain axon or neuron was significantly increased.

Interestingly, this hyperconnectivity was not specific to cBF-innervated cells, but was also evident for noncholinergic innervation, with mutant animals having a significantly higher number of GFP-expressing cells in the
mPFC than that observed in ChAT-cre control mice (Fig. 3a, b). Although the ChAT-positive cells in the basal forebrain were TdTomato-positive (Supplementary Figure S3b), a minority of cells were both TdTomato- and GFPpositive (Supplementary Figure S3c). However, about half of the TdTomato-labeled cells in the MPFC were also labeled by GFP, and this proportion did not change with 


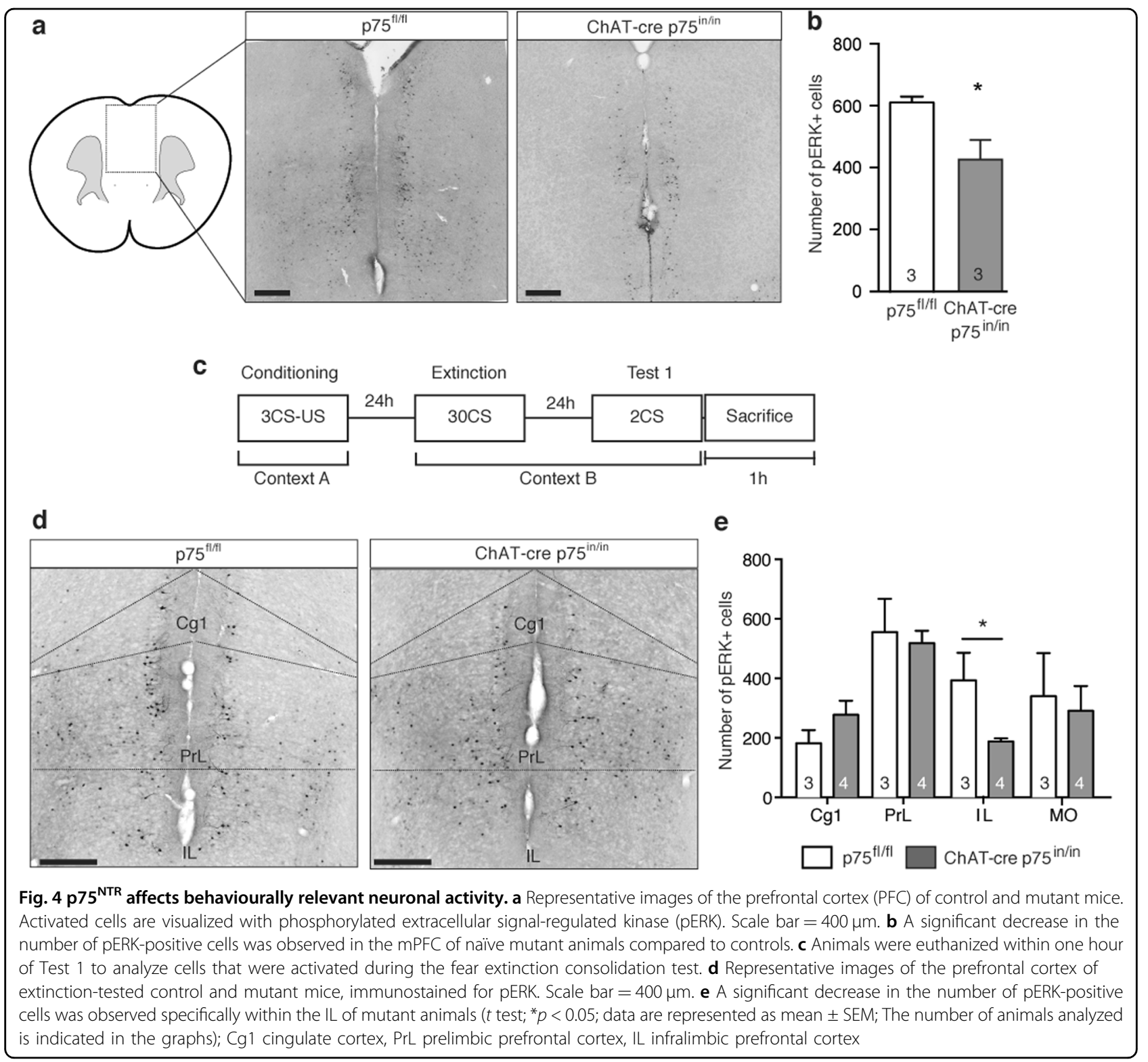

genotype (Supplementary Figure S3d). These results suggest that $\mathrm{p} 75^{\mathrm{NTR}}$ affects the synaptic connectivity of cholinergic neurons to the mPFC, and also indirectly influences non-cholinergic afferent synaptic innervation of neurons within this region.

In contrast to these results, no difference in the extent of either cholinergic or non-cholinergic innervation was seen in the hippocampus of the same animals, although many fewer rostral hippocampal neurons were labeled by the HSV129 virus than mPFC cells, despite the former being a larger target structure (Supplementary Figure $\mathrm{S} 3 \mathrm{e}-\mathrm{g}$ ). These data suggest that the role of $\mathrm{p} 75^{\mathrm{NTR}}$ and the nature of $\mathrm{cBF}$ neuronal innervation of postsynaptic neurons varies between target brain areas.
To determine whether restoring $\mathrm{p} 75^{\mathrm{NTR}}$ expression affected the synaptic hyperconnectivity, we injected the HSV129 virus into ChAT-cre $\mathrm{p} 75^{\mathrm{in} / \text { in }}$ and ChAT-cre control animals that had been previously injected with the p75ICD-GFP AAV. The expression of p75ICD-GFP had no significant effect on the extent of septohippocampal innervation in either case (Supplementary Figure S3e-g). However, there were significantly fewer TdTomatolabeled cholinergic cells in the mPFC of mutant animals in which the p75ICD-GFP was expressed compared to the number in ChAT-cre $\mathrm{p} 75^{\mathrm{in} / \mathrm{in}}$ animals injected with the control Tau-GFP AAV (Fig. 3a, c). Non-cholinergic synaptic innervation to the PFC was also significantly reduced in p75ICD-expressing mutant animals 
(Fig. 3e-g). The levels of both cholinergic and noncholinergic synaptic innervation in the ChAT-cre $\mathrm{p} 75^{\mathrm{in} / \mathrm{in}}$ + p75ICD-GFP animals were not significantly different from those in ChAT-cre control mice. This indicates that re-introduction of $\mathrm{p} 75^{\mathrm{NTR}}$ to adult $\mathrm{cBF}$ neurons restores mPFC synaptic connectivity back to wild-type levels, but does not affect septohippocampal innervation.

\section{Basal forebrain synaptic hyperinnervation to the PFC reduces postsynaptic activity within the MPFC}

We next asked whether postsynaptic neuronal activity was altered in the $\mathrm{mPFC}$ of $\mathrm{p} 75^{\mathrm{NTR}}$-deficient mice. The activation of the calcium-activated signaling pathway via the phosphorylated extracellular signal-regulated kinase (pERK) was assessed as pERK activity in neurons is observed immediately following cellular activity ${ }^{37}$. The number of pERK-positive cells in the mPFC was first determined for naïve $\mathrm{p} 75^{\mathrm{fl} / \mathrm{fl}}$ and ChAT-cre $\mathrm{p} 75^{\mathrm{in} / \mathrm{in}}$ animals, revealing a significant reduction in the p $75^{\mathrm{NTR}}$ deficient animals compared to controls (Fig. 4a, b). To explore whether the ERK activity correlated with neuronal function, the number of pERK-positive cells in MPFC was determined for mice euthanized within $1 \mathrm{~h}$ of fear extinction recall (Fig. 4c). The number of pERK-positive cells within the mPFC was significantly increased compared to that of naïve mice. Furthermore, although the number of pERK cels in the prelimbic, cingulate and medial orbital cortices did not differ between the two genotypes (Fig. $4 \mathrm{~d}-\mathrm{f}$ ), a significant decrease in the number of pERK-positive cells within the ILPFC, a nucleus of the mPFC that is critically involved in fear extinction consolidation $^{38}$, was observed in the ChAT-cre $\mathrm{p} 75^{\mathrm{in} / \mathrm{in}}$ animals compared to the $\mathrm{p} 75^{\mathrm{f} / \mathrm{fl}}$ controls (Fig. $4 \mathrm{~d}-\mathrm{f}$ ).

In order to determine which types of cells were activated in the mutant and control mice, we co-stained MPFC sections for pERK and GABAergic, glutamatergic or cholecystokinin (CCK) markers, namely parvalbumin, calcium/calmodulindependent protein kinase II (CaMKII) and CCK antibodies, respectively. pERK did not colocalize with either GABAergic or CCK markers (data not shown), whereas $\sim 15 \%$ of pERKpositive cells in the ILPFC of mice of both genotypes costained with the glutamatergic marker (Supplementary Figure S4a, b). As there were 50\% fewer pERK-positive cells in the ILPFC after extinction testing, these data indicate that the cBF synaptic hyperinnervation of the MPFC, which arises due to the loss of $\mathrm{p} 75^{\mathrm{NTR}}$ expression, reduces by half the number of principal glutamatergic neurons within the ILPFC that are activated following fear extinction recall, potentially explaining the behavioral change in the $\mathrm{p} 75^{\mathrm{NTR}}$-deficient mice.

\section{Discussion}

Here we demonstrate that loss of $\mathrm{p} 75^{\mathrm{NTR}}$ expression from $\mathrm{cBF}$ neurons induces synaptic hyperconnectivity within the mPFC and results in altered fear extinction consolidation. Re-expression of a constitutively active fragment of $\mathrm{p} 75^{\mathrm{NTR}}$ in the form of p75ICD-GFP in mPFCprojecting $\mathrm{cBF}$ neurons of adult ChAT-cre $\mathrm{p} 75^{\mathrm{in} / \mathrm{in}}$ mice not only restored fear extinction behavior, but also reversed the level of basal forebrain connectivity in the mPFC to that observed in control animals. These results indicate that adult $\mathrm{p} 75^{\mathrm{NTR}}$ signaling is important for regulating connectivity between basal forebrain and mPFC neurons, which in turn modulates fear extinction consolidation.

The major cellular phenotype of cBF neurons observed in ChAT-cre $\mathrm{p} 75^{\mathrm{in} / \mathrm{in}}$ mice was a $\sim 10$-fold increase in efferent connectivity to the $\mathrm{mPFC}$, as measured by transneuronal tracing. Interestingly, we did not find a corresponding increase in the axonal arborization of individual neurons projecting to either the mPFC or the hippocampus. Although an increase in the density of hippocampal innervation in a complete p $75^{\mathrm{NTR}}$-deficient mouse has been reported ${ }^{18,19}$, our previous study using conditional ChAT-cre $\mathrm{p} 75^{\mathrm{in} / \mathrm{in}}$ mice did not reproduce this, instead finding a significant increase in cBF axonal density specifically to layer $\mathrm{V}$ neurons in the barrel $\operatorname{cortex}^{16}$. Given that the methods used in the previous studies did not account for the $25 \%$ increase in cBF neuronal number, our current findings suggest that $\mathrm{p} 75^{\mathrm{NTR}}$ does not play a substantive role in regulating the extent of axonal arborization of individual $\mathrm{CBF}$ neurons, but rather regulates the number of synaptic connections that each cBF axon makes with its target(s). This would support the notion that the previous observations were a consequence of an increased number of neurons rather than changes to individual arbor sizes. In support of this interpretation, the expression of p75ICD-GFP had no discernable pruning effect on overall axonal innervation but reversed the synaptic hyperinnervation in the mPFC of mutant mice without affecting cBF neuron number. Although we can not rule out a subtle effect on axonal outgrowth due to p $75^{\mathrm{NTR}}$, a possible increase in axonal arbor size following p75ICD expression correlates with reduced cBF synapse density and therefore cannot be the explanation for our results.

The observed changes to the synaptic innervation patterns of basal forebrain neurons in the mPFC of ChATcre $\mathrm{p} 75^{\mathrm{in} / \mathrm{in}}$ animals could be due to mutant cells having increased release of the HSV129 virus, resulting in increased expression of postsynaptic HSV129 markers in these mice. However, this is unlikely given that the number of postsynaptic hippocampal cells labeled by HSV129 was not significantly different between control and knockout animals of the same cohort (Supplementary Figure S3e-g). Furthermore, we found a change in innervation patterns of both cholinergic and noncholinergic targets in the mPFC that could not be fully 
accounted for by local basal forebrain connectivity. Taken together, these findings suggest that the observed cellular phenotypes in mutant mice are not due to differences in the uptake or release of HSV129. Rather the observed changes to postsynaptic innervation in the mutant mice are specific to $\mathrm{mPFC}$-projecting $\mathrm{cBF}$ neurons due to the absence of $\mathrm{p} 75^{\mathrm{NTR}}$.

We were surprised by the finding that non-cholinergic basal forebrain innervation to the mPFC of $\mathrm{p} 75^{\mathrm{NTR}}$-deficient mice was altered, given that $\mathrm{p} 75^{\mathrm{NTR}}$ is only expressed by, and was genetically eliminated from, cBF neurons. However, the rescue of this phenotype by reexpression of $\mathrm{p} 75 \mathrm{ICD}-\mathrm{GFP}$ in ChAT-cre $\mathrm{p} 75^{\mathrm{in} / \mathrm{in}}$ mice indicates that the increase in non-cholinergic synaptic innervation was mediated by a cholinergic mechanism. Our tracing studies revealed that $\sim 50 \%$ of basal forebrain postsynaptic targets in the MPFC were co-labeled by both recombined HSV129 (derived from a cre-positive cell) and native HSV129 (derived from a cre-negative cell), suggesting that a significant proportion of mPFC neurons receive input from both cholinergic and non-cholinergic basal forebrain neurons. Analysis of co-expression in the basal forebrain revealed that a subset of tdTomatopositive cells co-expressed GFP, and expression at $72 \mathrm{~h}$ was not limited to ChAT-positive cells, suggestive of innervation of neighboring cells by cBF neurons. It has previously been shown that there is cross-talk between cell populations in the basal forebrain, with the activity of GABAergic cells of the basal forebrain driving the efflux of acetylcholine ${ }^{39}$, and GABA often being co-released with acetylcholine ${ }^{40}$. We therefore postulate that the observed cBF synaptic hyperinnervation in the absence of $\mathrm{p} 75^{\mathrm{NTR}}$ drives the recruitment of additional synaptic input from other cell and neurotransmitter types within the basal forebrain-mPFC circuit, which in turn influences the balance of cortical activity and output.

Despite the strong indication of hyperinnervation in the mPFC of ChAT-cre $\mathrm{p} 75^{\mathrm{in} / \mathrm{in}}$ mice, the synaptic innervation in the hippocampus of mutant animals of the same cohort was unchanged, and expression of the p75ICDGFP had no discernable effect in this region. Furthermore, despite robust labeling of axons by the Tau-GFP virus, there were many fewer HSV129-infected postsynaptic cells in the hippocampus compared to the number found in the mPFC. This suggests that septohippocampal basal forebrain neurons may exert their influence on hippocampal activity by volume rather than synaptic neurotransmission $^{7,41-43}$. This would explain why we failed to observe any changes in septohippocampal neuronal structure or hippocampal function in our previous ${ }^{16}$ or present study. Secondly, our results indicate that $\mathrm{p} 75^{\mathrm{NTR}}$ specifically regulates the point-to-point connectivity of neurons in the basal forebrain to their synaptically connected targets, while having little or no effect in areas where volume neurotransmission may predominate. However, future studies will need to address this further through electrophysiological assessments of hippocampal and cortical activity in the absence of $\mathrm{p} 75^{\mathrm{NTR}}$ in $\mathrm{cBF}$ neurons

Basal forebrain hyperinnervation to the $\mathrm{mPFC}$ reduced the level of basal neuronal activity in the MPFC as measured by pERK immunostaining. Whether these cells are less synaptically active or other signaling activity is reduced is unclear. However, the decrease in pERKpositive cells specifically within the ILPFC immediately after testing for fear extinction consolidation suggests a change in physiolgically relevant neuronal activity within this cortical region. It is widely accepted that the ILFPC plays a critical role in fear extinction, with activation of ILPFC glutamatergic neurons that project to the central amygdala being a key means by which fear-related responses are inhibited following extinction ${ }^{24-26,44,45}$. Therefore, the observed decrease in glutamatergic neuron pERK activity within the ILPFC of ChAT-cre $\mathrm{p} 75^{\mathrm{in} / \mathrm{in}}$ animals is consistent with the observed deficit in extinction consolidation in the mutant mice.

The expression of p75ICD-GFP within the medial septum cBF neurons of the $\mathrm{p} 75^{\mathrm{NTR}}$-deficient mice both reversed the mPFC synaptic hyperinnervation and rescued fear extinction behavior. These data strongly link the p $75^{\mathrm{NTR}}$-mediated synaptic changes within the fear extinction circuit to the observed behavioral change. Several other reports suggest that loss of $\mathrm{p} 75^{\mathrm{NTR}}$ can affect the synaptic function of neurons both directly and indirectly ${ }^{3,17,46,47}$. Our study cannot resolve whether basal forebrain hyperinnervation of the ILPFC directly underlies reduced ILPFC neuronal activity, or whether hyperinnervation to other mPFC nuclei and/or other cortical areas indirectly influences ILPFC activity during fear extinction consolidation and testing. For example, we have previously demonstrated that ChAT-cre $\mathrm{p} 75^{\text {in/in }}$ mice have better spatial memory in an allothetic (uncued) place avoidance spatial memory task ${ }^{16}$. Therefore, it is possible that the altered extinction is due to enhanced recall of the CS-US pairing ${ }^{43}$. Similarly, acetylcholine is released during associative learning ${ }^{2}$, and the enhanced fear recall in the p $75^{\mathrm{NTR}}$-deficient mice could be due to altered context-dependent associations. Regardless of the mechanism, our study provides compelling evidence that successful consolidation of fear extinction memories requires coordinated activity between cholinergic and non-cholinergic neurons in the basal forebrain and glutamatergic neurons within the $\mathrm{mPFC}$, and demonstrates that this process is controlled by $\mathrm{p} 75^{\mathrm{NTR}}$-mediated signaling.

In summary, this work has highlighted that the nature of $\mathrm{cBF}$ neuronal innervation of postsynaptic neurons varies between target areas and has revealed that $\mathrm{cBF}$ 
synaptic connectivity in the adult is regulated by $\mathrm{p} 75^{\mathrm{NTR}}$ signaling. We have further determined a role for medial septal $\mathrm{CBF}$ neurons projecting to the $\mathrm{mPFC}$ in the regulation of fear extinction. As such, cholinergic or neurotrophic modulators could be considered as candidate therapeutic targets for enhancing memory or, alternatively, for the treatment of conditions such as posttraumatic stress disorder.

\section{Acknowledgements}

This work was supported by the National Health and Medical Research Council of Australia (Project Grant 1049236 to EJC) and the Clem Jones Centre for Aging Dementia Research. Z.B. and MTT were supported by an Australian Postgraduate Awards and a University of Queensland Advantage Scholarship and a Australia Dementia Research Foundation Top-Upscholarship respectively. We thank the staff of the University of Queensland Biological Resources Facility for breeding and maintaining the animals used in this study, members of the Coulson laboratory past and present for helpful discussions and Rowan Tweedale for editorial assistance.

\section{Author details}

'Centre for Ageing Dementia Research, The University of Queensland, Brisbane, QLD 4072, Australia. ${ }^{2}$ Queensland Brain Institute, The University of Queensland, Brisbane, QLD 4072, Australia. ${ }^{3}$ Faculty of Medicine, School of Biomedical Sciences, The University of Queensland, Brisbane, QLD 4072, Australia. ${ }^{4}$ Department of Pharmacology, University of Oxford, Oxford, UK

\section{Conflict of interest}

The authors declare that they have no conflict of interest.

\section{Publisher's note}

Springer Nature remains neutral with regard to jurisdictional claims in published maps and institutional affiliations.

Supplementary Information accompanies this paper at (https://doi.org/ 10.1038/s41398-018-0248-x).

Received: 2 May 2018 Accepted: 14 May 2018

Published online: 21 September 2018

\section{References}

1. Zaborszky L., van den Pol A., Gyengesi E. in The Mouse Nervous System (eds Watson, C., Paxinos, G. \& Puelles, L.) Elsevier, USA 684-718 (2012).

2. Ballinger Elizabeth, C., Ananth, M., Talmage David, A. \& Role Lorna, W. Basal forebrain cholinergic circuits and signaling in cognition and cognitive decline. Neuron 91, 1199-1218 (2016).

3. Gibon, J. et al. proBDNF and p75NTR control excitability and persistent firing of cortical pyramidal neurons. J. Neurosci. 35, 9741-9753 (2015).

4. Hasselmo, M. E., Bodelon, C. \& Wyble, B. P. A proposed function for hippocampal theta rhythm: separate phases of encoding and retrieval enhance reversal of prior learning. Neural Comput. 14, 793-817 (2002).

5. Hasselmo, M. E. \& Schnell, E. Laminar selectivity of the cholinergic suppression of synaptic transmission in rat hippocampal region CA1: computational modeling and brain slice physiology. J. Neurosci. 14, 3898-3914 (1994).

6. Gulledge, A. T., Park, S. B., Kawaguchi, Y. \& Stuart, G. J. Heterogeneity of phasic cholinergic signaling in neocortical neurons. J. Neurophysiol. 97, 2215-2229 (2007).

7. Chen, N., Sugihara, H. \& Sur, M. An acetylcholine-activated microcircuit drives temporal dynamics of cortical activity. Nat. Neurosci. 18, 892-902 (2015).

8. Higley, M. J., Soler-Llavina, G. J. \& Sabatini, B. L. Cholinergic modulation of multivesicular release regulates striatal synaptic potency and integration. Nat. Neurosci. 12, 1121-1128 (2009).

9. Gu, Z., Lamb, P. W. \& Yakel, J. L. Cholinergic coordination of presynaptic and postsynaptic activity induces timing-dependent hippocampal synaptic plasticity. J. Neurosci. 32, 12337-12348 (2012).
10. Conner, J. M., Chiba, A. A. \& Tuszynski, M. H. The basal forebrain cholinergic system is essential for cortical plasticity and functional recovery following brain injury. Neuron 46, 173-179 (2005).

11. Conner, J. M., Culberson, A., Packowski, C., Chiba, A. A. \& Tuszynski, M. H. Lesions of the basal forebrain cholinergic system impair task acquisition and abolish cortical plasticity associated with motor skill learning. Neuron 38, 819-829 (2003).

12. Higley, M. J. \& Picciotto, M. R. Neuromodulation by acetylcholine: Examples from schizophrenia and depression. Curr. Opin. Neurobiol. 29, 88-95 (2014).

13. Wallace, T. L. \& Bertrand, D. Importance of the nicotinic acetylcholine receptor system in the prefrontal cortex. Biochem. Pharmacol. 85, 1713-1720 (2013).

14. Douchamps, V. \& Mathis, C. A second wind for the cholinergic system in Alzheimer's therapy. Behav. Pharmacol. 28(2 and 3-Spec Issue), 112-123 (2017).

15. Dokter, M. et al. Implications of p75NTR for dentate gyrus morphology and hippocampus-related behavior revisited. Brain Struct. \&Funct. 220, 1449-1462 (2014).

16. Boskovic, Z. et al. The role of p75NTR in cholinergic basal forebrain structure and function. J. Neurosci. 34, 13033-13038 (2014).

17. Barrett, G. L. et al. Enhanced spatial memory and hippocampal long-term potentiation in p75 neurotrophin receptor knockout mice. Hippocampus $\mathbf{2 0}$ 145-152 (2010).

18. Greferath, U. et al. Enlarged cholinergic forebrain neurons and improved spatial learning in p75 knockout mice. Eur. J. Neurosci. 12, 885-893 (2000).

19. Yeo, T. T. et al. Absence of p75NTR causes increased basal forebrain cholinergic neuron size, choline acetyltransferase activity, and target innervation. J. Neurosci. 17, 7594-7605 (1997).

20. Knox, D. The role of basal forebrain cholinergic neurons in fear and extinction memory. Neurobiol. Learn. Mem. 133, 39-52 (2016).

21. Jiang, L. et al. Cholinergic signaling controls conditioned fear behaviors and enhances plasticity of cortical-amygdala circuits. Neuron 90, 1057-1070 (2016).

22. Knox, D. \& Keller, S. M. Cholinergic neuronal lesions in the medial septum and vertical limb of the Diagonal Bands of Broca induce contextual fear memory generalization and impair acquisition of fear extinction. Hippocampus $\mathbf{2 6}$ 718-726 (2015)

23. Maren, S. Neurobiology of Pavlovian fear conditioning. Annu. Rev. Neurosci. 24 897-931 (2001).

24. Maren, S., Phan, K. L. \& Liberzon, I. The contextual brain: implications for fear conditioning, extinction and psychopathology. Nat. Rev. Neurosci. 14, 417-428 (2013).

25. Milad, M. R. \& Quirk, G. J. Fear extinction as a model for translational neuroscience: ten years of progress. Annu. Rev. Psychol. 63, 129-151 (2012).

26. Lin, Q. et al. The brain-specific microRNA miR-128b regulates the formation of fear-extinction memory. Nat. Neurosci. 14, 1115-1117 (2011).

27. Rossi, J. et al. Melanocortin-4 receptors expressed by cholinergic neurons regulate energy balance and glucose homeostasis. Cell Metab. 13, 195-204 (2011).

28. Atasoy, D., Aponte, Y., Su, H. H. \& Sternson, S. M. A FLEX switch targets Channelrhodopsin-2 to multiple cell types for imaging and long-range circuit mapping. J. Neurosci. 28, 7025-7030 (2008).

29. McClure, C., Cole, K. L., Wulff, P., Klugmann, M. \& Murray, A. J. Production and titering of recombinant adeno-associated viral vectors. J. Vis. Exp. 57, e3348 (2011).

30. McGovern, A. E. et al. Distinct brainstem and forebrain circuits receiving tracheal sensory neuron inputs revealed using a novel conditional anterograde transsynaptic viral tracing system. J. Neurosci. 35, 7041-7055 (2015).

31. Matusica, D. et al. An intracellular domain fragment of the $p 75$ neurotrophin receptor (p75NTR) enhances TrkA receptor function. J. Biol. Chem. 288, 11144-11154 (2013)

32. Skeldal, S., Matusica, D., Nykjaer, A. \& Coulson, E. J. Proteolytic processing of the p75 neurotrophin receptor: A prerequisite for signalling?: Neuronal life, growth and death signalling are crucially regulated by intra-membrane proteolysis and trafficking of p75(NTR). BioEssays 33, 614-625 (2011).

33. Kenchappa, R. S. et al. Ligand-dependent cleavage of the P75 neurotrophin receptor is necessary for NRIF nuclear translocation and apoptosis in sympathetic neurons. Neuron 50, 219-232 (2006).

34. Ceni, C. et al. The p75NTR intracellular domain generated by neurotrophininduced receptor cleavage potentiates Trk signaling. J. Cell Sci. 123(Pt 13), 2299-2307 (2010).

35. Bloem, B. et al. Topographic mapping between basal forebrain cholinergic neurons and the medial prefrontal cortex in Mice. J. Neurosci. 34, 16234-16246 (2014). 
36. Lo, L. \& Anderson, D. J. A Cre-dependent, anterograde transsynaptic viral tracer for mapping output pathways of genetically marked neurons. Neuron $\mathbf{7 2}$, 938-950 (2011).

37. Ha, S. \& Redmond, L. ERK mediates activity dependent neuronal complexity via sustained activity and CREB-mediated signaling. Dev. Neurobiol. 68, 1565-1579 (2008)

38. Milad, M. R. \& Quirk, G. J. Neurons in medial prefrontal cortex signal memory for fear extinction. Nature 420, 70-74 (2002).

39. Roland, J. J. et al. Medial Septum-Diagonal Band of Broca (MSDB) GABAergic regulation of hippocampal acetylcholine efflux is dependent on cognitive demands. J. Neurosci. 34, 506-514 (2014).

40. Tritsch, N. X., Granger, A. J. \& Sabatini, B. L. Mechanisms and functions of GABA co-release. Nat. Rev. Neurosci. 17, 139-145 (2016).

41. Verhoog, M. B. et al. Layer-specific cholinergic control of human and mouse cortical synaptic plasticity. Nat. Commun. 7, 12826 (2016)
42. Anaclet, $C$. et al. Basal forebrain control of wakefulness and cortical rhythms. Nat. Commun. 6, 8744 (2015).

43. Goard, M. \& Dan, Y. Basal forebrain activation enhances cortical coding of natural scenes. Nat. Neurosci. 12, 1444-1449 (2009).

44. Do-Monte, F. H., Manzano-Nieves, G., Quinones-Laracuente, K., Ramos-Medina, L. \& Quirk, G. J. Revisiting the role of infralimbic cortex in fear extinction with optogenetics. J. Neurosci. 35, 3607-3615 (2015).

45. Marek, R., Strobel, C., Bredy, T. W. \& Sah, P. The amygdala and medial prefrontal cortex: partners in the fear circuit. J. Physiol. 591(Pt 10), 2381-2391 (2013).

46. Woo, N. H. et al. Activation of p75NTR by proBDNF facilitates hippocampal long-term depression. Nat. Neurosci. 8, 1069-1077 (2005).

47. Rosch, H., Schweigreiter, R., Bonhoeffer, T., Barde, Y. A. \& Korte, M. The neurotrophin receptor p75NTR modulates long-term depression and regulates the expression of AMPA receptor subunits in the hippocampus. Proc. Natl Acad. Sci. USA 102, 7362-7367 (2005). 